\title{
A diet high in sugar-sweetened beverage and low in fruits and vegetables is associated with adiposity and a pro-inflammatory adipokine profile
}

\author{
Corinna Koebnick ${ }^{1 *}$, Mary Helen Black ${ }^{1}$, Jun Wu ${ }^{1}$, Yu-Hsiang Shu ${ }^{1}$, Adrienne W. MacKay ${ }^{2}$, \\ Richard M. Watanabe $2,3,4$, Thomas A. Buchanan ${ }^{3,5}$ and Anny H. Xiang ${ }^{1}$ \\ ${ }^{1}$ Department of Research E Evaluation, Kaiser Permanente Southern California, Pasadena, CA, USA \\ ${ }^{2}$ Department of Preventive Medicine, Keck School of Medicine, University of Southern California, Los Angeles, CA, USA \\ ${ }^{3}$ USC Diabetes and Obesity Research Institute, University of Southern California, Los Angeles, CA, USA \\ ${ }^{4}$ Department of Physiology E Biophysics, Keck School of Medicine, University of Southern California, Los Angeles, CA, USA \\ ${ }^{5}$ Department of Medicine, Keck School of Medicine, University of Southern California, Los Angeles, CA, USA
}

(Submitted 7 March 2018 - Final revision received 17 July 2018 - Accepted 19 July 2018 - First published online 30 October 2018)

\section{Abstract}

Diet, obesity and adipokines play important roles in diabetes and CVD; yet, limited studies have assessed the relationship between diet and multiple adipokines. This cross-sectional study assessed associations between diet, adiposity and adipokines in Mexican Americans. The cohort included 1128 participants (age $34.7 \pm 8.2$ years, BMI $29.5 \pm 5.9 \mathrm{~kg} / \mathrm{m}^{2}, 73.2 \%$ female). Dietary intake was assessed by $12-\mathrm{month}$ food frequency questionnaire. Adiposity was measured by BMI, total percentage body fat and percentage trunk fat using dual-energy X-ray absorptiometry. Adiponectin, apelin, C-reactive protein (CRP), dipeptidyl peptidase- 4 (DPP-IV), IL- $1 \beta$, IL-1ra, IL-6, IL-18, leptin, lipocalin, monocyte chemo-attractant protein-1 (MCP-1), resistin, secreted frizzled protein 4 (SFRP-4), SFRP-5, TNF- $\alpha$ and visfatin were assayed with multiplex kits or ELISA. Joint multivariate associations between diet, adiposity and adipokines were analysed using canonical correlations adjusted for age, sex, energy intake and kinship. The median (interquartile range) energy intake was 9514 $(7314,11912) \mathrm{kJ} / \mathrm{d}$. Overall, $55 \%$ of total intake was accounted for by carbohydrates (24\% from sugar). A total of $66 \%$ of the shared variation between diet and adiposity, and $34 \%$ of diet and adipokines were explained by the top canonical correlation. The diet component was most represented by sugar-sweetened beverages (SSB), fruit and vegetables. Participants consuming a diet high in SSB and low in fruits and vegetables had higher adiposity, CRP, leptin, and MCP-1, but lower SFRP-5 than participants with high fruit and vegetable and low SSB intake. In Mexican Americans, diets high in SSB but low in fruits and vegetables contribute to adiposity and a pro-inflammatory adipokine profile.

Key words: Fruit: Vegetables: Sugar-sweetened beverages: Adipokines: Adiposity

Diet is one of the most important modifiable risk factor for obesity, type 2 diabetes and $\mathrm{CVD}^{(1)}$. Excess dietary intake and low energy expenditure through lack of physical activity lead to a positive energy balance and accumulation of excess fat in adipose tissue $^{(2,3)}$. Although the primary function of adipose tissue is to store excess nutrients as TAG and to release NEFA during fasting, it also secretes a large amount of hormones and signalling molecules $^{(4,5)}$. These adipose tissue products are collectively referred to as adipokines and may act as mediators between dietary pattern and disease risk ${ }^{(6-8)}$.

Adipokines may play an important role in the pathogenesis of complications of obesity such as type 2 diabetes, metabolic syndrome and $\mathrm{CVD}^{(9-14)}$. Obesity is associated with up-regulation of pro-inflammatory and down-regulation of anti-inflammatory adipokines, which promote and ameliorate insulin resistance, respectively ${ }^{(15,16)}$. Some adipokines also directly influence $\beta$-cell apoptosis and insulin secretion ${ }^{(17)}$. Anti-inflammatory adipokines counteract these effects ${ }^{(17)}$. Lower adiponectin and rising C-reactive protein (CRP) are associated with declining $\beta$-cell function in women at high risk for type 2 diabetes ${ }^{(18)}$. In metaanalyses and reviews, associations between diet, dietary pattern and adipokines were demonstrated for adiponectin ${ }^{(19)}, \mathrm{CRP}^{(19-21)}$ and IL- $6^{(19)}$. On the basis of these studies, several dietary inflammation indices were constructed to reflect biological pathways mediating inflammation ${ }^{(22,23)}$. However, our understanding of dietary intake on broader adipokine profile remains limited.

The goal of the present study was to address this knowledge gap by examining the relationship between the consumption of

Abbreviations: CCA, canonical correlation analysis; CRP, C-reactive protein; DPP-IV, dipeptidyl peptidase-4; GDM, gestational diabetes mellitus; HighSSB/ LowFV, high in sugar-sweetened beverages and low in fruit and vegetables; LowSSB/HighFV, low in sugar-sweetened beverages and high in fruit and vegetables; MCP-1, monocyte chemo-attractant protein-1; SFRP, secreted frizzled protein; SSB, sugar-sweetened beverages. 
major food groups, three measures of adiposity and a large set of adipokines in a Mexican American cohort enriched with individuals at high risk for type 2 diabetes. The findings from our study help identify the most influential dietary drivers of obesity and adipokine levels, which in turn may be used to elucidate the primary dietary components that contribute to metabolic dysfunction in Mexican Americans.

\section{Methods \\ Study design and subjects}

Participants of this study were from the BetaGene study, a family-based study of obesity, insulin resistance and $\beta$-cell dysfunction in Mexican Americans ${ }^{(24)}$. Study design and recruitment has been previously described ${ }^{(24)}$. Probands were individuals with the condition of interest for the BetaGene study seeking medical attention at Los Angeles County/University of Southern California Medical Center, the Kaiser Permanente Southern California health plan membership, and obstetric/ gynaecologic clinics at local southern California hospitals (online Supplementary Fig. S1). Inclusion criteria for all probands were a Mexican-American ancestry (both parents and at least three grandparents Mexican or of Mexican descent) and one of two general family structures: (a) at least two siblings and three first cousins from a single nuclear family or (b) at least five siblings available for study participation. All participants are either (1) probands with a diagnosis of gestational diabetes mellitus (GDM) within 5 years of enrolment of the study, (2) probands with normal glucose levels (non-GDM) during a pregnancy within the 5 years of enrolment of the study, or (3) siblings or first cousins of probands. Non-GDM probands were age, BMI and parity-matched to the GDM probands.

Participants were excluded if they showed evidence of $\beta$-cell autoimmunity by glutamic acid decarboxylase- 65 testing or if they had fasting glucose $>7 \cdot 0 \mathrm{mmol}$, as acute insulin response to glucose is often absent above this level. Final study participants were probands, or siblings and first cousins of probands who completed the baseline screening ( $n$ 1585). For the present study, we further excluded participants who did not meet the inclusion criteria after baseline screening $(n$ 101) and those with incomplete visits ( $n$ 231) or data ( $n$ 91), resulting in a final analytical cohort of 1128 participants.

Data were collected in two separate visits to the General Clinical Research Center at the University of Southern California. The first visit consisted of a physical examination, dietary and physical activity questionnaires, a fasting blood draw for lipid measurements and sample storage for adipokine measurement, as previously described ${ }^{(25-27)}$. Non-GDM probands and family members of probands with fasting glucose $<7.0 \mathrm{mmol} / 1$ were invited for a second visit as previously described and dual-energy X-ray absorptiometry scan was performed to assess their total body fat and trunk fat ${ }^{(28)}$. A total of 1128 participants from 403 families (ranging from one to twelve participants per family) completed the dietary assessment, and had all adiposity (BMI, dual-energy X-ray absorptiometry scan) and adipokine measures.

\section{Ethics}

All protocols for BetaGene were approved by the Institutional Review Boards of participating institutions, and all participants provided written informed consent before participation. The study is registered under NCT03301519.

\section{Dietary assessment}

Assessment of dietary intake for this cohort has been previously reported ${ }^{(29)}$. The food consumption for the past 12 months was assessed using the 126-item semi-quantitative Harvard food frequency questionnaire $(\mathrm{FFQ})^{(30)}$. Trained bilingual interviewers administered the FFQ to all participants. The FFQ consisted of a list of foods with a standardised serving size and a selection of nine frequency categories ranging from never or $<1$ serving per month to $>6$ servings per day during the past year. An openended free-text section was used to capture food items that did not appear on the standard list and included information on usual serving size and number of servings consumed per week for incorporation in the dietary intake calculations for each subject. The frequency of consumption of each food from the FFQ was multiplied by the specified portion size to calculate the food consumption in servings per day, and then converted to grams per day. FFQs with reported energy intakes $>3$ sDs from the mean were considered invalid and excluded from analysis $(n 37)^{(29)}$. Food items were grouped into dairy products (low- and high-fat items such as cheese, milk, yoghurt, ice cream, cream cheese and butter), fruits (raw, cooked, fresh, canned, frozen or dehydrated fruits), fruit juices, meat (fresh and processed meats such as beef, lamb, pork, poultry), fish (including other seafood), starchy foods (pasta, rice, grains, bread, potatoes, etc.), sugarsweetened beverages (SSB) (caffeinated colas, caffeine-free colas, other carbonated SSB and non-carbonated SSB such as fruit punches, lemonades or others) and vegetables (raw, cooked, fresh, canned, frozen or dehydrated vegetables and legumes).

\section{Body mass and composition}

Height and weight were measured using a beam medical scale and wall-mounted stadiometer to the nearest $0.1 \mathrm{~kg}$ and $0.1 \mathrm{~cm}$, respectively. BMI was calculated as body weight $(\mathrm{kg}) /$ height $(\mathrm{m})^{2}$. Total body fat mass and trunk fat mass were determined by dualenergy X-ray absorptiometry (Discovery-W ${ }^{\mathrm{TM}}$ DEXA system).

\section{Adipokines}

Fasting plasma samples were used to measure adipokines. Adiponectin, IL-1 $\beta$, IL-6, leptin, lipocalin, monocyte chemoattractant protein-1 (MCP-1), that is chemokine ligand 2, or CCL2, resistin and TNF- $\alpha$ were assayed using two Millipore multiplex kits with magnetic bead panels (Millipore). Assay sensitivity for adiponectin, lipocalin and resistin are 11, 1.7 and $2 \cdot 2 \mathrm{pg} / \mathrm{ml}$, respectively. Intra- and inter-assay variations are $<10$ and $<15 \%$ respectively, and accuracy ranged from 87 to $91 \%$ for all adipokines in the panel. Sensitivities for leptin, IL-1 $\beta$, IL-6, MCP- 1 and TNF- $\alpha$ are 19.0, 0.4, 0.2, 1.3 and $0.3 \mathrm{pg} / \mathrm{ml}$, respectively. Intra- and inter-assay variations are $<15 \%$ and accuracy 
is $>90 \%$ for all adipokines in the panel. ELISA was used to measure CRP (Millipore), apelin, dipeptidyl peptidase-4 (DPP-IV), IL-18, visfatin (Ray Biotech), IL-1ra (AssayBiotech), secreted frizzled protein 4 (SFRP-4) and SFRP-5 (USCN Life Science). Assay sensitivity for CRP, apelin, DPP-IV, IL-18, visfatin, IL-1ra, SFRP-4 and SFRP-5 is: $0.0002 \mu \mathrm{g} / \mathrm{ml}, 36.55 \mathrm{pg} / \mathrm{ml}, 0.261 \mathrm{ng} /$ $\mathrm{ml}, 6 \cdot 1 \mathrm{pg} / \mathrm{ml}, 13.5 \mathrm{pg} / \mathrm{ml}, 2.4 \mathrm{pg} / \mathrm{ml}, 28.3 \mathrm{pg} / \mathrm{ml}$ and $0.39 \mathrm{pg} / \mathrm{ml}$. Intra- and inter-assay variations are $<10$ and $<15 \%$, respectively, for all adipokines. Accuracy was $>95 \%$ for most adipokines; accuracy for SFRP-4 and SFRP-5 ranged from 85 to $95 \%$.

The proportion of participants with measures below the limit of detection was less than $2 \%$ for IL-6, MCP-1, TNF- $\alpha$, IL- $1 \beta$, SFRP-5, apelin and IL-1ra, whereas those for IL- $1 \beta$ and IL-18 reached 7 and $14 \%$, respectively. Values of these adipokines that were lower than the limit of detection were imputed using the square root of the limit of detection ${ }^{(31)}$.

\section{Statistical analysis}

The cohort characteristics are described by medians and corresponding 25th and 75th percentiles. The consumption of major food groups, measures of adiposity and adipokines were log-transformed to approximate univariate normality where appropriate before data analyses. Correlation among the consumption of the major food groups, between each of the major food groups and measures of adiposity, and among adipokines were estimated after adjustment for age and sex.

Canonical correlation analysis (CCA) was used to assess the multivariate associations between the food groups and adiposity (BMI, percentage of body fat and trunk fat), as well as food groups and adipokines. CCA is a multivariate analysis of crosscorrelations among two sets of quantities where each set has multiple variables. CCA finds linear combinations of each set that have the maximum correlation with each other ${ }^{(32,33)}$. The loading coefficient represents each variable's contribution to the specific set being investigated. To appropriately account for relatedness among individuals owing to family structure in this study, linear mixed-effect kinship models were used and CCA analyses were performed on the kinship-adjusted covariance of food group consumption $v$. adiposity, and food group consumption $v$. adipokines after adjustment for age, sex and total energy intake ${ }^{(34)}$. The top canonical correlation was displayed by a heliograph where the bar length is an indicator for the strength of association for each variable to the top linear component identified in $\mathrm{CCA}^{(35)}$.

To characterise the sample from the CCA results, we grouped the cohort into three groups based on participant's consumption of the top dietary components identified from CCA analyses relative to the tertile distribution of the whole cohort. Measures of adiposity and adipokines were compared among the three groups after adjusting for age, sex, total energy intake and kinship using the mixed-effects model $^{(34)}$. To visualise the impact of dietary group on different adipokines relative to each other, we applied a standardisation method to remove the measurement units associated with each adipokine and displayed the standardised residuals by dietary groups after adjusting for age, sex, total energy intake and kinship. All statistical analyses were conducted using SAS version 9.3 (SAS Institute, Inc.) and R v.3.3.0 (36).
Table 1. Characteristics of BetaGene study participants (Medians and 25th, 75th percentiles; percentages)

\begin{tabular}{|c|c|c|}
\hline & \multicolumn{2}{|c|}{ BetaGene participants $(n 1128$} \\
\hline & Median & 25th, 75th percentiles \\
\hline \multicolumn{3}{|l|}{ Demographic characteristics } \\
\hline Females (\%) & 73.2 & \\
\hline Age (years) & 34.4 & $29 \cdot 2,39.6$ \\
\hline \multicolumn{3}{|l|}{ Dietary factors } \\
\hline Total energy (kJ/d) & 9514 & 7314,11912 \\
\hline Total carbohydrate (\% of total energy) & 55.4 & $49 \cdot 5,61 \cdot 2$ \\
\hline Sugars ( $\%$ of total energy) & 23.6 & $19 \cdot 2,28.0$ \\
\hline Fat ( $\%$ of total energy) & $28 \cdot 7$ & $24 \cdot 4,32 \cdot 6$ \\
\hline Protein (\% of total energy) & $17 \cdot 3$ & $15 \cdot 4,19 \cdot 3$ \\
\hline Dairy products $(g / d)$ & 281.4 & $154.7,402 \cdot 9$ \\
\hline Fish $(g / d)$ & 36.5 & $23 \cdot 0,58 \cdot 5$ \\
\hline Fruits $(g / d)$ & 240.6 & $146 \cdot 7,365 \cdot 3$ \\
\hline Fruit juices $(\mathrm{ml} / \mathrm{d})$ & $142 \cdot 2$ & $48 \cdot 3,252 \cdot 1$ \\
\hline Meat $(\mathrm{g} / \mathrm{d})$ & $177 \cdot 4$ & $123 \cdot 4,235.3$ \\
\hline Starchy foods $(\mathrm{g} / \mathrm{d})$ & $190 \cdot 4$ & $133.0,254.5$ \\
\hline Sugar-sweetened beverages $(\mathrm{ml} / \mathrm{d})$ & 346.9 & $137 \cdot 1,497 \cdot 3$ \\
\hline Vegetables $(\mathrm{g} / \mathrm{d})$ & 385.5 & $277 \cdot 9,544.0$ \\
\hline \multicolumn{3}{|l|}{ Body composition } \\
\hline BMl $\left(\mathrm{kg} / \mathrm{m}^{2}\right)$ & $28 \cdot 7$ & $25 \cdot 4,32 \cdot 6$ \\
\hline Total body fat (\%) & 35.9 & $29 \cdot 0,40 \cdot 6$ \\
\hline Trunk fat $(\%)$ & $12 \cdot 9$ & $9 \cdot 7,17 \cdot 1$ \\
\hline \multicolumn{3}{|l|}{ Adipokines } \\
\hline Adiponectin ( $\mu \mathrm{g} / \mathrm{ml})$ & 10.49 & $7 \cdot 23,16 \cdot 87$ \\
\hline Apelin $(\mathrm{ng} / \mathrm{ml})$ & $1 \cdot 14$ & $0 \cdot 71,1 \cdot 70$ \\
\hline C-reactive protein $(\mu \mathrm{g} / \mathrm{ml})$ & 1.44 & $0.61,3.18$ \\
\hline DPP-IV $(\mathrm{ng} / \mathrm{ml})$ & 265.04 & $210.19,339.59$ \\
\hline $\mathrm{IL}-1 \beta(\mathrm{pg} / \mathrm{ml})$ & 0.63 & $0.49,0.88$ \\
\hline IL-1ra (pg/ml) & 11.47 & $6.96,18.07$ \\
\hline $\mathrm{IL}-6(\mathrm{pg} / \mathrm{ml})$ & 2.85 & $1.70,4.95$ \\
\hline IL-18 (pg/ml) & 173.63 & $60.18,300.64$ \\
\hline Leptin (ng/ml) & 14.09 & $6 \cdot 73,24 \cdot 82$ \\
\hline Lipocalin (ng/ml) & 63.17 & $52.50,76.44$ \\
\hline MCP-1 (pg/ml) & $107 \cdot 12$ & $84 \cdot 17,132 \cdot 28$ \\
\hline Resistin (ng/ml) & $18 \cdot 15$ & $14 \cdot 31,23 \cdot 66$ \\
\hline SFRP-4 (ng/ml) & 75.03 & $53 \cdot 47,106.85$ \\
\hline SFRP-5 (ng/ml) & 14.82 & $9 \cdot 41,22 \cdot 75$ \\
\hline TNF- $a(\mathrm{pg} / \mathrm{ml})$ & $2 \cdot 87$ & $2.11,3.98$ \\
\hline Visfatin (ng/ml) & 13.69 & $10.58,17.83$ \\
\hline
\end{tabular}

DPP-IV, dipeptidyl peptidase-4; MCP-1, monocyte chemo-attractant protein-1; SFRP, secreted frizzled protein.

\section{Results}

\section{Characteristics of BetaGene participants}

Participants of the BetaGene study ( $n$ 1128) were $34 \cdot 7 \pm 8 \cdot 2$ years of age, mostly female (73.2\%) and had a median BMI of $28.7 \mathrm{~kg} / \mathrm{m}^{2}$ (interquartile range, $25 \cdot 4,32 \cdot 6$ ) and total body fat of $35 \cdot 9 \%$ (29.0-40.6) (Table 1). The BetaGene proband generation included 338 probands (30.0\%), 527 siblings (46.7\%) and 263 first cousins $(23.3 \%)$. The majority of participants were overweight or obese ( $n 887,79 \%$ ). The median (interquartile range) energy intake was $9514(7314,11912) \mathrm{kJ} / \mathrm{d}$. Although $55.4 \%$ of total energy intake that accounted for was by carbohydrates, $23.6 \%$ was derived from sugar. The energy intake from fat was $28.7 \%$, and $17.3 \%$ from protein. Participants consumed a median (interquartile range) of $385.5(277.99,544.0) \mathrm{g} / \mathrm{d}$ vegetables, $240 \cdot 6(146 \cdot 7,365 \cdot 3) \mathrm{g} / \mathrm{d}$ fruits, $142 \cdot 2(48 \cdot 3,252 \cdot 1) \mathrm{ml} / \mathrm{d}$ fruit juices and $346 \cdot 9(137 \cdot 1,497 \cdot 3) \mathrm{ml} / \mathrm{d}$ SSB (Table 1). 
Table 2. Age-, sex and kinship-adjusted correlations between consumption of major food groups, body composition and adipokines

\begin{tabular}{|c|c|c|c|c|c|c|c|c|}
\hline & Dairy products & Fish & Fruits & Fruit juice & Meat & Starchy foods & SSB & Vegetables \\
\hline \multicolumn{9}{|l|}{ Food consumption } \\
\hline Dairy products & - & & & & & & & \\
\hline Fish & $0.095^{\star}$ & - & & & & & & \\
\hline Fruits & $0.253^{*}$ & $0.229^{*}$ & - & & & & & \\
\hline Fruit juice & $0.103^{*}$ & $0.111^{*}$ & $0.263^{\star}$ & - & & & & \\
\hline Meat & $0.084^{*}$ & $0.133^{*}$ & $0.066^{\star}$ & 0.043 & - & & & \\
\hline Starchy foods & $0.121^{\star}$ & $0.184^{\star}$ & $0.168^{*}$ & $0.154^{\star}$ & $0.354^{*}$ & - & & \\
\hline SSB & 0.000 & 0.040 & $-0.093^{*}$ & $0.088^{*}$ & $0.219^{*}$ & $0 \cdot 210^{*}$ & - & \\
\hline Vegetables & $0.176^{\star}$ & $0 \cdot 265^{*}$ & $0.369^{*}$ & $0.175^{\star}$ & $0.247^{*}$ & $0.260^{\star}$ & -0.018 & - \\
\hline \multicolumn{9}{|l|}{ Body composition } \\
\hline BMI $\left(\mathrm{kg} / \mathrm{m}^{2}\right)$ & -0.056 & 0.006 & -0.046 & $-0.077^{\star}$ & 0.003 & $-0.084^{\star}$ & $0.076^{*}$ & -0.018 \\
\hline Total body fat (\%) & -0.051 & -0.006 & $-0.100^{*}$ & $-0.093^{*}$ & 0.010 & $-0.077^{\star}$ & $0.101^{*}$ & -0.038 \\
\hline Trunk fat (\%) & -0.049 & -0.001 & $-0.075^{\star}$ & $-0.104^{\star}$ & 0.001 & $-0.084^{\star}$ & $0.079^{*}$ & -0.008 \\
\hline \multicolumn{9}{|l|}{ Adipokines } \\
\hline Adiponectin & 0.003 & -0.021 & 0.026 & 0.032 & -0.030 & 0.011 & -0.053 & 0.011 \\
\hline Apelin & -0.058 & -0.044 & -0.036 & $-0.093^{*}$ & 0.046 & 0.002 & 0.054 & -0.013 \\
\hline C-reactive protein & 0.025 & 0.025 & -0.054 & -0.038 & 0.033 & -0.051 & $0.085^{\star}$ & -0.027 \\
\hline DPP-IV & 0.028 & -0.016 & 0.049 & 0.029 & -0.012 & 0.022 & -0.005 & 0.024 \\
\hline $\mathrm{IL}-1 \beta$ & 0.039 & 0.046 & -0.043 & -0.018 & -0.028 & 0.035 & -0.005 & -0.012 \\
\hline IL-1ra & 0.005 & -0.034 & -0.049 & -0.025 & 0.013 & -0.016 & 0.027 & -0.016 \\
\hline IL-6 & -0.001 & 0.033 & -0.031 & 0.010 & 0.001 & 0.023 & 0.025 & -0.015 \\
\hline IL-18 & -0.021 & -0.041 & $-0.062^{*}$ & -0.026 & 0.024 & 0.009 & 0.058 & -0.010 \\
\hline Leptin & -0.047 & 0.016 & $-0.089^{\star}$ & $-0.059^{\star}$ & 0.021 & $-0.073^{*}$ & $0.093^{*}$ & -0.055 \\
\hline Lipocalin & 0.001 & 0.005 & 0.015 & 0.054 & 0.027 & 0.005 & 0.040 & -0.012 \\
\hline MCP-1 & 0.032 & -0.010 & $-0.059^{\star}$ & -0.013 & 0.042 & 0.008 & $0.090^{*}$ & $-0.061^{*}$ \\
\hline Resistin & 0.009 & 0.001 & -0.028 & 0.029 & 0.001 & 0.009 & 0.023 & -0.033 \\
\hline SFRP-4 & 0.028 & 0.008 & 0.009 & 0.003 & -0.023 & -0.027 & 0.052 & -0.037 \\
\hline SFRP-5 & 0.012 & 0.040 & 0.033 & 0.022 & $-0.051^{*}$ & -0.002 & $-0.070^{*}$ & 0.004 \\
\hline TNF- $a$ & 0.023 & 0.019 & -0.055 & -0.037 & -0.005 & 0.001 & $0.056^{\star}$ & $-0.076^{\star}$ \\
\hline Visfatin & 0.033 & $0.063^{*}$ & 0.006 & 0.026 & -0.022 & -0.004 & 0.030 & -0.032 \\
\hline
\end{tabular}

SSB, sugar-sweetened beverages; DPP-IV, dipeptidyl peptidase-4; MCP-1, monocyte chemo-attractant protein-1; SFRP, secreted frizzled protein.

${ }^{*}$ Correlations are significant at $P \leq 0.05$.

\section{Food consumption and body composition}

All measures of adiposity (BMI, total percentage body fat and percentage trunk fat) were negatively correlated with the consumption of fruits and fruit juice, marginally with vegetables and starchy foods, and positively correlated with SSB (Table 2).

In multivariate analysis using CCA on variables adjusted for sex, age, energy intake and kinship, a total of $66 \%$ of the shared variation between diet and measures of adiposity was explained by the top linear association between the two $(P<0 \cdot 001)$ with loading coefficients for food consumption and body composition given in Table 3. No other canonical component explained the association between the two $(P>0.05)$. The strongest predictors of adiposity were a higher consumption of SSB (canonical loading $\rho=0.73)$ as well as a lower consumption of fruit $(\rho=-0.45)$ and fruit juices $(\rho=-0 \cdot 46)$. The consumption of meat, dairy products and starchy foods showed a modest to low association $(\rho \leq 0.30)$ with measures of adiposity. The top canonical correlation is shown as heliograph in Fig. 1(a).

\section{Food consumption and adipokines}

In partial correlations adjusted for sex, age and kinship, several food groups were correlated with adipokine levels (Table 2).

A higher consumption of SSB was significantly associated with higher CRP, leptin, MCP-1, SRFP- 4 and TNF- $\alpha$. Fruit consumption was negatively correlated with CRP, IL- $1 \beta$, IL-1ra and leptin and positively with DPP-IV. Fruit juice consumption was negatively correlated with apelin and leptin. A higher vegetable consumption was associated with lower leptin, MCP-1, SRFP-4 and TNF- $\alpha$. A higher meat consumption was associated with lower SRFP-5 and a higher consumption of starchy foods with lower leptin.

In multivariate analysis using CCA on variables adjusted for sex, age, energy intake and kinship, a total of $33 \%$ of the shared variation between diet and adipokines was explained by the top linear association between the two $(P=0 \cdot 017$; Table 3$)$. No other components explained the association between the two $(P>0.05)$. The top dietary component was most represented by SSB (canonical loading $\rho=0.74)$, fruits $(\rho=-0.54)$, fruit juices $(\rho=-0.42)$ and vegetables $(\rho=-0 \cdot 39)$, whereas the adipokine component was most represented by leptin $(\rho=0 \cdot 65)$, CRP $(\rho=0 \cdot 55)$, MCP-1 $(\rho=0.52)$, apelin $(\rho=0 \cdot 40)$, SRFP-5 $(\rho=-0.34)$, adiponectin $(\rho=-0 \cdot 32)$ and IL-18 $(\rho=0 \cdot 30)$. All other canonical loading factors were $<0 \cdot 30$. The top canonical correlation is shown as heliograph in Fig. 1(b). To assess the role of adiposity in the multivariate association between diet and adipokines, we repeated the CCA analysis by further adjusting for total body fat. Adjusting for adiposity removed the shared variation between diet and adipokines and the top linear association between the two was no longer significant $(P=0 \cdot 36)$.

\section{Adiposity and adipokines by tertiles of the top dietary components from canonical correlation analysis results}

CCA analysis identified SSB, fruit, fruit juice and vegetable as the top dietary components that were associated with adiposity 
Table 3. Joint multivariate canonical loadings between food consumption, measures of adiposity and adipokines

\begin{tabular}{|c|c|c|c|c|}
\hline & \multicolumn{4}{|c|}{ Canonical loadings } \\
\hline & \multicolumn{2}{|c|}{ Diet $v$. adiposity } & \multicolumn{2}{|c|}{ Diet $v$. adipokines } \\
\hline & Model $1^{*}$ & Model 2† & Model $1^{*}$ & Model $2 \dagger$ \\
\hline Canonical correlation & 0.185 & 0.176 & 0.221 & 0.220 \\
\hline Proportion of variance explained (\%) & 68.9 & $66 \cdot 0$ & $33 \cdot 8$ & 33.4 \\
\hline$P$ & $<0.001$ & $<0.001$ & 0.019 & 0.017 \\
\hline \multicolumn{5}{|l|}{ Food consumption } \\
\hline Dairy products & -0.26 & -0.14 & -0.06 & -0.02 \\
\hline Fish & -0.03 & 0.09 & -0.03 & -0.03 \\
\hline Fruits & $-0.55 \ddagger$ & $-0.45 \ddagger$ & $-0.54 \ddagger$ & $-0.54 \ddagger$ \\
\hline Fruit juices & $-0.55 \ddagger$ & $-0.46 \ddagger$ & $-0.44 \ddagger$ & $-0.42 \ddagger$ \\
\hline Meat & 0.02 & 0.26 & 0.20 & 0.29 \\
\hline Starchy foods & $-0.44 \ddagger$ & -0.27 & -0.24 & -0.21 \\
\hline Sugar-sweetened beverages & $0.51 \ddagger$ & $0.73 \ddagger$ & $0.66 \ddagger$ & $0.74 \ddagger$ \\
\hline Vegetables & $-0 \cdot 16$ & 0.00 & $-0.40 \ddagger$ & $-0.39 \ddagger$ \\
\hline \multicolumn{5}{|l|}{ Body composition } \\
\hline BMI & $0.83 \ddagger$ & $0.82 \ddagger$ & & \\
\hline Total body fat & $0.99 \ddagger$ & $0.99 \ddagger$ & & \\
\hline Trunk fat & $0.95 \ddagger$ & $0.94 \ddagger$ & & \\
\hline \multicolumn{5}{|l|}{ Adipokines } \\
\hline Adiponectin & & & $-0.32 \ddagger$ & $-0.32 \ddagger$ \\
\hline Apelin & & & $0.39 \ddagger$ & $0.40 \ddagger$ \\
\hline C-reactive protein & & & $0.55 \ddagger$ & $0.55 \ddagger$ \\
\hline DPP-IV & & & -0.15 & -0.14 \\
\hline $\mathrm{IL}-1 \beta$ & & & -0.01 & -0.02 \\
\hline IL-1ra & & & 0.13 & 0.14 \\
\hline IL-6 & & & 0.00 & -0.01 \\
\hline IL-18 & & & $0.30 \ddagger$ & $0.30 \ddagger$ \\
\hline Leptin & & & $0.67 \ddagger$ & $0.65 \ddagger$ \\
\hline Lipocalin & & & 0.06 & 0.08 \\
\hline MCP-1 & & & $0.50 \ddagger$ & $0.52 \ddagger$ \\
\hline Resistin & & & 0.08 & 0.09 \\
\hline SFRP-4 & & & 0.21 & 0.21 \\
\hline SFRP-5 & & & $-0.32 \ddagger$ & $-0.34 \ddagger$ \\
\hline TNF- $a$ & & & 0.29 & 0.28 \\
\hline Visfatin & 0.12 & 0.11 & & \\
\hline
\end{tabular}

DPP-IV, dipeptidyl peptidase-4; MCP-1, monocyte chemo-attractant protein-1; SFRP, secreted frizzled protein.

${ }^{*}$ Model 1: Adjusted for age, sex and kinship.

† Model 2: Adjusted for age, sex, kinship and total energy intake.

$\ddagger$ Loadings $\geq 0.30$.

and/or adipokines. Next, we classified participants as 'High' intake if their consumptions were at or greater than the third tertile of the distribution, and 'Low' intake if were at or lower than the first tertile of the distribution. Three groups were formed: (1) 'High' in SSB but 'Low' in fruit (including fruit juice) and vegetable consumption (HighsSB/LowFV), (2) 'Low' in SSB but 'High' in fruit (including fruit juice) and vegetable consumption (LowSSB/HighFV) and (3) the remaining combinations as 'Other'. Table 4 presents the sex, age, total energy intake and kinship-adjusted adipokines by the three dietary groups. Participants consuming a LowSSB/HighFV diet had lower BMI, total percentage body fat, percentage trunk fat than participants consuming a HighSSB/LowFV diet. Participants consuming a HighSSB/LowFV diet had higher CRP, leptin, apelin and MCP-1, but lower SFRP-5 and adiponectin than participants consuming a LowSSB/HighFV diet, although the differences in adiponectin and apelin were not statistically significant. Participants consuming a HighSSB/LowFV diet also had higher BMI, total percentage body fat, percentage trunk fat than participants consuming a LowSSB/HighFV diet. Standardisation revealed that the differences in anti-inflammatory adipokines between HighSSB/LowFV and LowSSB/HighFV were most pronounced for SFRP-5, whereas the differences in pro-inflammatory adipokines was most pronounced for leptin and MCP-1 (Fig. 2).

\section{Discussion}

In this cross-sectional analysis in Mexican Americans with a high prevalence of overweight or obesity, high SSB and low fruit and vegetable intake was identified as the main dietary factors associated with increased adiposity. A high consumption of SSB but low fruit and vegetable intake also correlated with a pro-inflammatory adipokine profile characterised by increased CRP, leptin and MCP-1, and decreased SFRP-5 compared with a low SSB but high fruit and vegetable intake. The association between dietary intake and adipokine profile was primarily explained by adiposity rather than an independent association between diet and adipokines.

The novelty of this study is the use of a multivariate analysis approach accounting for collinearity to assess the multivariate 
(a)

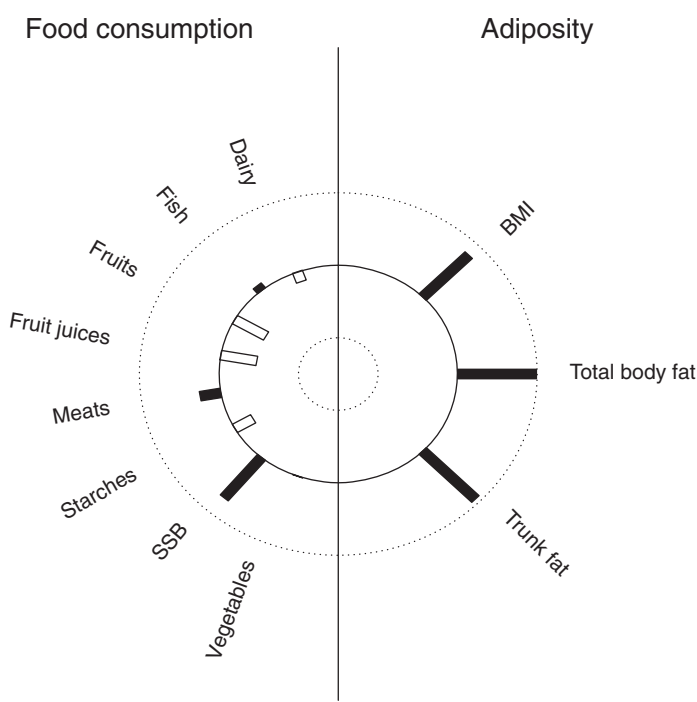

(b)

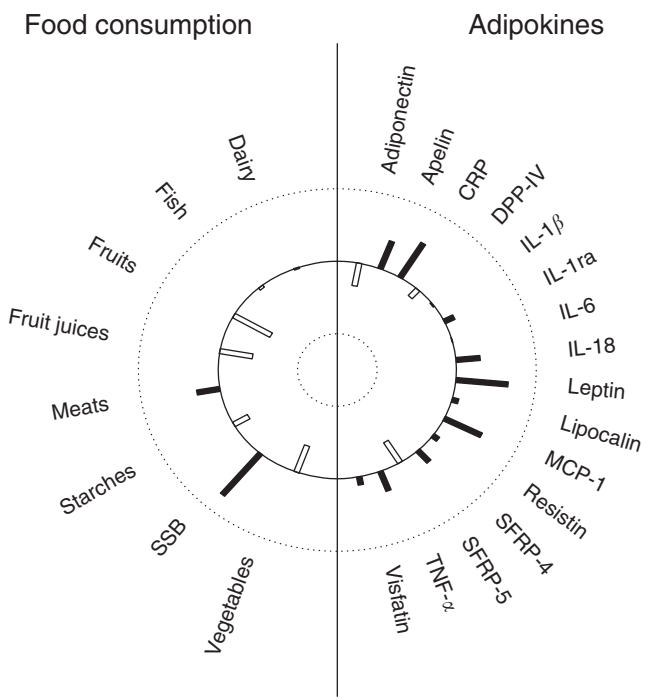

Fig. 1. Canonical-correlation heliograph demonstrating the joint multivariate association between food consumption and adiposity ((a) explained variation $66 \%$, $P<0.001)$ as well as food consumption and adipokines ((b) explained variation $33 \%, P=0.017)$ after adjusting for sex, age, energy intake and kinship: dark $\mathrm{bar}=$ positive association, white bar = negative association; bar length = strength of association ranging from 1 (outer circle) to -1 (inner circle). SSB, sugar-sweetened beverages. CRP, C-reactive protein; DPP-IV, dipeptidyl peptidase-4; MCP-1, monocyte chemo-attractant protein-1; SFRP, secreted frizzled protein.

Table 4. Adipokine concentrations in participants consuming a diet which was (1) high in sugar-sweetened beverages (SSB) and low in fruit and vegetables (HighSSB/LowFV), (2) low in SSB and high in fruit and vegetables (LowSSB/HighFV) or (3) any other consumption (Geometric or arithmetic means with their standard errors)

\begin{tabular}{|c|c|c|c|c|c|c|c|}
\hline \multirow[b]{2}{*}{ Variables* } & \multicolumn{2}{|c|}{ HighSSB/LowFV (A, $n$ 147) } & \multicolumn{2}{|c|}{ Other ( $n$ 834) } & \multicolumn{2}{|c|}{ LowSSB/HighFV (B, $n$ 147) } & \multirow[b]{2}{*}{$P(\mathrm{~A} v . \mathrm{B})$} \\
\hline & Mean & SE & Mean & SE & Mean & SE & \\
\hline \multicolumn{8}{|l|}{ Adiposity } \\
\hline BMI $\left(\mathrm{kg} / \mathrm{m}^{2}\right)$ & $29 \cdot 8$ & $29 \cdot 3,30 \cdot 2$ & 28.9 & $28 \cdot 7,29 \cdot 2$ & $28 \cdot 2$ & $27 \cdot 8,28 \cdot 6$ & 0.007 \\
\hline Body fat (\%) & 35.8 & $35 \cdot 4,36 \cdot 3$ & 34.8 & $34 \cdot 6,35 \cdot 0$ & 33.4 & $33 \cdot 0,33.9$ & $<0.001$ \\
\hline Trunk fat $(\mathrm{kg})$ & 13.6 & $13 \cdot 1,14 \cdot 1$ & $12 \cdot 4$ & $12 \cdot 2,12 \cdot 7$ & 11.7 & $11 \cdot 2,12 \cdot 1$ & 0.001 \\
\hline \multicolumn{8}{|l|}{ Adipokines } \\
\hline Adiponectin $(\mu \mathrm{g} / \mathrm{ml})$ & $10 \cdot 29$ & $9.78,10.83$ & 11.23 & $10.94,11.52$ & 11.64 & $11 \cdot 06,12 \cdot 25$ & 0.074 \\
\hline Apelin $(\mathrm{ng} / \mathrm{ml})$ & 1.13 & $1.06,1.20$ & 1.10 & $1.07,1.12$ & 1.00 & $0.94,1.06$ & 0.15 \\
\hline C-reactive protein $(\mu \mathrm{g} / \mathrm{ml})$ & 1.55 & $1.41,1.70$ & 1.42 & $1.36,1.49$ & $1 \cdot 13$ & $1.03,1.25$ & 0.017 \\
\hline DPP-IV (ng/ml) & $269 \cdot 17$ & $261 \cdot 24,277 \cdot 34$ & 266.62 & $262.98,270.32$ & 273.73 & $265 \cdot 71,282.00$ & 0.68 \\
\hline $\mathrm{IL}-1 \beta(\mathrm{pg} / \mathrm{ml})$ & 0.73 & $0.70,0.77$ & 0.71 & $0.69,0.73$ & 0.69 & $0.66,0.73$ & 0.40 \\
\hline IL-1ra (pg/ml) & 12.55 & $11 \cdot 79,13 \cdot 37$ & $11 \cdot 10$ & $10 \cdot 79,11.43$ & $10 \cdot 54$ & $9 \cdot 90,11 \cdot 22$ & 0.045 \\
\hline $\mathrm{IL}-6(\mathrm{pg} / \mathrm{ml})$ & 2.95 & $2.73,3.19$ & 2.95 & $2.86,3.05$ & 2.59 & $2 \cdot 39,2.80$ & 0.23 \\
\hline IL-18 (pg/ml) & $122 \cdot 51$ & $108.40,138.45$ & 111.81 & $105.99,117.96$ & 89.93 & $79.60,101.59$ & 0.071 \\
\hline Leptin (ng/ml) & 13.86 & $12 \cdot 99,14.78$ & $12 \cdot 14$ & $11.76,12.53$ & $10 \cdot 39$ & $9 \cdot 74,11.08$ & 0.001 \\
\hline Lipocalin (ng/ml) & $62 \cdot 41$ & $60.87,63.98$ & $62 \cdot 33$ & $61.58,63.09$ & 63.69 & $62 \cdot 13,65 \cdot 28$ & 0.55 \\
\hline MCP-1 (pg/ml) & $120 \cdot 16$ & $116 \cdot 70,123.62$ & 111.60 & $110.06,111.31$ & $106 \cdot 50$ & $103.05,109.94$ & 0.005 \\
\hline Resistin (ng/ml) & 19.01 & $18.31,19.75$ & 18.73 & $18.40,19.07$ & $18 \cdot 70$ & $18.01,19.42$ & 0.75 \\
\hline SFRP-4 (ng/ml) & $75 \cdot 11$ & $72 \cdot 19,78 \cdot 14$ & 76.03 & $74.55,77.55$ & $72 \cdot 27$ & $69 \cdot 48,75 \cdot 18$ & 0.47 \\
\hline SFRP-5 (ng/ml) & $16 \cdot 33$ & $15 \cdot 42,17 \cdot 24$ & 17.55 & $17.09,18.01$ & $19 \cdot 30$ & $18 \cdot 39,20 \cdot 20$ & 0.015 \\
\hline TNF- $a(\mathrm{pg} / \mathrm{ml})$ & 3.09 & $2 \cdot 95,3.24$ & 2.76 & $2 \cdot 70,2 \cdot 82$ & 2.57 & $2.45,2.70$ & 0.006 \\
\hline Visfatin $(\mathrm{ng} / \mathrm{ml})$ & 14.07 & $13.56,14.60$ & 13.99 & $13.75,14.23$ & $14 \cdot 22$ & $13.71,14.75$ & 0.84 \\
\hline
\end{tabular}

DPP-IV, dipeptidyl peptidase-4; MCP-1, monocyte chemo-attractant protein-1; SFRP, secreted frizzled protein.

* Data are geometric means (except BMI, MCP-1 and SFRP-5, which are given as arithmetic means) and standard errors adjusted for sex, age and total energy intake based on linear mixed-effect kinship models.

association between diet, three measures of adiposity (i.e. BMI, total body fact percentage and trunk fat percentage) and a large set of adipokines. Adipokines were chosen because each adipokine has previously shown association with energy homeostasis and $\beta$-cell function; these also include adipokines that are less studied in humans, such as apelin, MCP-1 and SFRP-5. We identified that high SSB and low fruit and vegetable intake were the main common food groups associated with increased adiposity and pro-inflammatory adipokine profiles. Obesity may be the pathway linking the association between diet and adipokines. Obesity is a disorder of the energy homeostasis system; the mechanisms to regulate and defend excess body weight are currently not well understood and involve several adipokines $^{(37,38)}$. Our results are consistent with previous evidence 


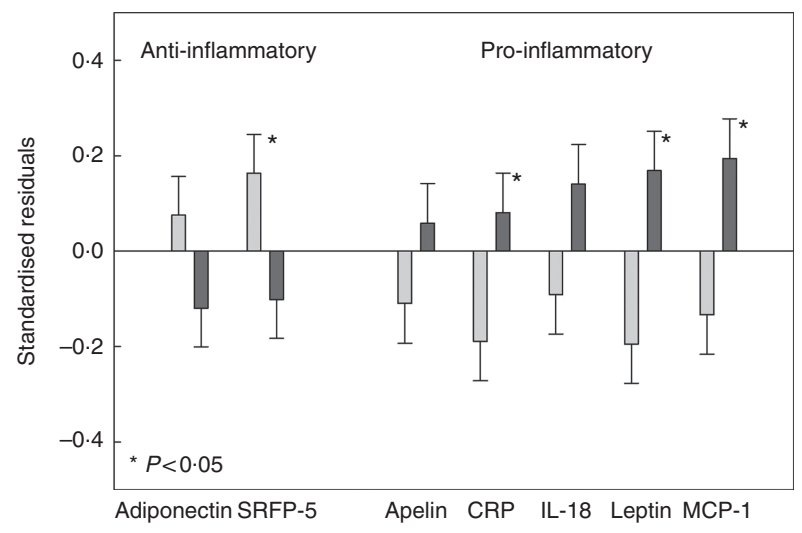

Fig. 2. Standardised residuals after adjusting for sex, age and energy intake of selected adipokines of participants consuming a diet which was high in sugarsweetened beverages (SSB) and low in fruit, fruit juices and vegetables (HighSSB/LowFV, $n$ 147) compared with participants consuming a diet which was low in sugar-sweetened beverages and high in fruit, fruit juices and vegetables (LowSSB/HighFV, $n$ 147). Adipokines shown exhibited loadings $\geq 0.30$ in canonical correlation analysis. The standardisation was to remove measurement units to better picture the impact of dietary group on different adipokines relative to each other. $P$ values comparing HighSSB/LowFV and LowSSB/HighFV are provided from linear mixed-effect kinship models. $\square$, LowSSB/HighFV; $\square$, HighSSB/LowFV. CRP, C-reactive protein; MCP-1, monocyte chemo-attractant protein-1.

that obesity is generally characterised by up-regulation of proinflammatory and down-regulation of anti-inflammatory adipokines. We adjusted for total energy intake in our analysis. Our finding that adiposity was associated with high SSB and low fruit and vegetable intake independent of total energy intake is consistent with previous findings that well-balanced diets rich in fruit and vegetables but low in sugar, such as the Dietary Approaches to Stop Hypertension (DASH) or a Mediterranean diet, are associated with lower adiposity and morbidity ${ }^{(39-43)}$

It is concerning that the consumption of SSB in the USA has steadily increased over the past 30 years, and is particularly high among Mexican Americans ${ }^{(44,45)}$. A recent systematic review and meta-analyses of prospective cohort studies and randomised controlled trials strongly suggests that the consumption of SSB promotes weight gain in children and adults ${ }^{(46,47)}$, and increases the risk of metabolic syndrome and diabetes ${ }^{(48)}$. In the present study, BetaGene participants consumed an average of $347 \mathrm{ml}$ or approximately $12 \mathrm{fl} \mathrm{oz}$ of SSB per day. The mechanisms linking SSB and adiposity are not entirely clear, but it has been speculated that SSB can lead to weight gain through several different pathways independent of high-added sugar content. For example, consumption of SSB may result in an incomplete compensatory reduction in energy intake at subsequent meals after intake of liquid energy, and/or unregulated hepatic uptake and metabolism of fructose, which in turn leads to liver lipid accumulation, dyslipidaemia, decreased insulin sensitivity and increased uric acid levels ${ }^{(46,49)}$. On the contrary, a diet rich in fruits and vegetables has been shown to be associated with lower adiposity and less weight gain ${ }^{(50)}$. In one study, an increase in the intake of fruits and vegetables reduced the long-term risk of obesity and weight gain among middleaged women $^{(51)}$. The mechanisms for the inverse association between fruit and vegetable intakes and body weight may include a higher intake of dietary fibre and polyphenols as wells as greater satiety ${ }^{(52-54)}$.

Our understanding of the complex regulation of proinflammatory adipokines under varying dietary patterns remains limited. Studies investigating a wider spectrum of food groups, nutrients or adipokines are scant and the effects of fruits, vegetables and SSB on the regulation of adipokines are not well defined ${ }^{(19-23)}$. In the present study, the main correlates of a diet with high SSB and low fruit and vegetable intake were increased CRP, leptin and MCP-1, and decreased SFRP-5, which is consistent with metabolic dysfunction as found in obesity ${ }^{(17,55,56)}$.

Leptin, an adipokine that regulates energy balance, has been widely studied with regard to dietary associations and has often been measured with the pro-inflammatory marker $\mathrm{CRP}^{(17,57-59)}$. Leptin has an important role in regulation of glucose homeostasis, independent of its actions on food intake or body weight ${ }^{(60)}$. In a feedback loop, insulin stimulates leptin secretion from adipose tissue, while leptin inhibits insulin biosynthesis and secretion $^{(60)}$. Insulin resistance is associated with hyperleptinaemia and selective leptin resistance in individuals who are obese ${ }^{(61)}$. Leptin plays a crucial role in inflammatory pathways and increases the secretion of TNF- $\alpha$, IL- 6 and MCP- ${ }^{(62)}$. Human CRP is correlated with increased adiposity and plasma leptin. Circulating CRP binds to leptin, attenuates its physiological functions and may contribute to leptin resistance ${ }^{(57)}$. A high consumption of fruits and vegetables has been associated with lower CRP in several $^{(21,63-70)}$ but not in all studies ${ }^{(64,71-74)}$. The effects reported may be explained by their effect on energy balance and body weight $^{(63,64,68,75)}$. In two studies, the association between the consumption of fruits and vegetables with lower CRP became insignificant after adjusting for adiposity ${ }^{(64,70)}$. In several other studies, the results were not adjusted for adiposity ${ }^{(21,63,68)}$. The results from the present study indicated that a diet low in fruits and vegetables but high in SSB is associated with higher leptin and CRP but this association is mainly through adiposity.

In addition to these findings, we identified substantial contributions of SSB and fruit/vegetable consumption to levels of MCP-1 and SFRP-5. MCP-1 is one of the key chemokines regulating the migration and infiltration of monocytes and macrophages ${ }^{(55,76)}$ and drives obesity-related inflammation and insulin resistance ${ }^{(60)}$. An increase in adipocyte mass is related to an increase in MCP-1, which in turn increases the production of other pro-inflammatory adipokines such as IL- 6 and TNF- $\alpha$, leading to a down-regulation of adiponectin, decreased glucose uptake in muscle cells and a metabolism favouring glycogenolysis and gluconeogenesis ${ }^{(60,76)}$. Systemic insulin resistance, lipid accumulation in the liver and hepatic insulin resistance form are the consequences ${ }^{(77)}$. Although circulating levels of MCP-1 are increased in overweight and obese individuals ${ }^{(78,79)}$, little is known about the association between dietary factors and MCP-1. In a small study of young Taiwanese women, a daily tomato juice supplementation resulted in reduced MCP-1 but did not affect leptin concentrations $^{(80)}$. In mice, a high-fat diet increased the expression of MCP- ${ }^{(81)}$. Another recent study indicates that MCP-1 may play a key role in hyperglycaemia-induced inflammation that was alleviated after short-term insulin therapy in newly diagnosed patients with type 2 diabetes ${ }^{(82)}$. We previously reported that increasing adiposity contributed to a worsening imbalance of pro- and anti- 
inflammatory adipokines over time, but the canonical correlation between adiposity and MCP-1 was small ${ }^{(83)}$. Results from the present study suggest that a high consumption of SSB and low consumption of fruit and vegetables lead to increased MCP-1 concentrations. Future studies have to reveal if this association is mediated by obesity or if MCP-1 has a direct role in upregulating inflammation in response to diet

SRFP-5 is an adipokine with possible insulin sensitising and anti-inflammatory properties which leads to improved metabolic function in white adipose tissue cells ${ }^{(56)}$. SRFP-5 is an inhibitor of the Wnt signalling pathway, which is an important factor in energy balance regulation ${ }^{(84)}$. In a recent study, recombinant SRFP-5 significantly alleviated the expression of pro-inflammatory adipokines (IL- $1 \beta$, IL- 6 , TNF- $\alpha$ and MCP-1) in the liver of a mouse model with induced non-alcoholic fatty liver disease ${ }^{(85)}$, suggesting a link between SRFP-5 and MCP-1 consistent with findings from the present study. However, studies on its role in humans are scant and results are somewhat conflicting ${ }^{(84,86)}$. No studies could be identified linking a healthy dietary pattern to an increase in SRFP-5. However, energy restriction and weight loss were associated with improved insulin sensitivity and an increase in SRFP-5 in adults ${ }^{(87)}$ and children ${ }^{(88)}$ with obesity. In the present study, a high consumption of fruit and vegetables was associated with higher SRFP-5, and a high SSB consumption with lower SRFP-5 concentrations.

There are several strengths to this study. The large cohort of Mexican Americans without type 2 diabetes allowed us to identify significant dietary drivers of a wide range of pro- and anti-inflammatory adipokines, which has not been available in the literature to date. Use of CCA allowed us to capture the underlying multivariate correlation of multiple adipokines and multiple dietary factors to better understand their joint relationships while accounting for the collinearity among them. Our detailed measures of adiposity allowed us to investigate the association between dietary factors, adiposity and adipokines as well as to assess the role of adiposity in the relationship between diet and adipokines. The dietary factors represent the most important food groups and the adipokines were chosen to reflect relevant pro- and anti-inflammatory markers associated with $\beta$-cell dysfunction $(4,6,7,13,17,89,90)$. Our study provides a unique insight in the complex joint relationship between diet, adiposity and adipokines.

Several potential limitations should be mentioned. The crosssectional design of the present analyses precluded us from assessing the impact of changes in dietary factors on changes in adiposity and adipokine profiles over time. Our results demonstrate associations, so no causal conclusions can be made. We acknowledge that using a single dietary assessment may not account appropriately for within-subject variability. However, the FFQ has been validated and the food-based measures of dietary factors were reasonably accurate, although the FFQ tends to over-represent socially desirable foods ${ }^{(91)}$. Finally, results from this cohort of predominantly female Mexican Americans with a high prevalence of overweight and obesity may not be generalisable to other populations.

In conclusion, a diet high in SSB and low in fruits and vegetables may be associated with increased adiposity and a pro-inflammatory adipokine profile characterised by higher leptin, CRP and MCP-1 and lower anti-inflammatory SFRP-5 in Mexican Americans compared with a diet low in SSB and high in fruits and vegetables. Additional longitudinal studies, in a variety of populations, are warranted to understand the longterm implications and underlying mechanisms.

\section{Acknowledgements}

The authors thank the families who participated in the BetaGene Study and also acknowledge the efforts of our recruiting and technical staff.

This work was supported by grants R01-DK-100302 and R01-DK-061628 from the National Institute of Diabetes and Digestive and Kidney Diseases (NIDDK), and by grant UL1TR-001845 from the National Center for Advancing Translational Science, National Institutes of Health (NIH).

The authors have no potential conflicts of interest relevant to this article.

\section{Supplementary material}

For supplementary material/s referred to in this article, please visit https://doi.org/10.1017/S0007114518002726

\section{References}

1. Hruby A, Manson JE, Qi L, et al. (2016) Determinants and consequences of obesity. Am J Public Health 106, 1656-1662.

2. Pemberton VL, McCrindle BW, Barkin S, et al. (2010) Report of the National Heart, Lung, and Blood Institute's Working Group on obesity and other cardiovascular risk factors in congenital heart disease. Circulation 121, 1153-1159.

3. Rocha VZ \& Libby P (2009) Obesity, inflammation, and atherosclerosis. Nat Rev Cardiol 6, 399-409.

4. Abranches MV, Oliveira FC, Conceicao LL, et al. (2015) Obesity and diabetes: the link between adipose tissue dysfunction and glucose homeostasis. Nutr Res Rev 28, 121-132.

5. Jung UJ \& Choi MS (2014) Obesity and its metabolic complications: the role of adipokines and the relationship between obesity, inflammation, insulin resistance, dyslipidemia and nonalcoholic fatty liver disease. Int J Mol Sci 15, 6184-6223.

6. Ko BJ, Park KH \& Mantzoros CS (2014) Diet patterns, adipokines, and metabolism: where are we and what is next? Metabolism 63, 168-177.

7. Sanchez D, Miguel M \& Aleixandre A (2012) Dietary fiber, gut peptides, and adipocytokines. J Med Food 15, 223-230.

8. Silva FM, de Almeida JC \& Feoli AM (2011) Effect of diet on adiponectin levels in blood. Nutr Rev 69, 599-612.

9. Feakins RM (2016) Obesity and metabolic syndrome: pathological effects on the gastrointestinal tract. Histopathology $\mathbf{6 8}$, 630-640.

10. Saltevo J, Vanhala M, Kautiainen H, et al. (2008) Gender differences in C-reactive protein, interleukin-1 receptor antagonist and adiponectin levels in the metabolic syndrome: a population-based study. Diabet Med 25, 747-750.

11. DeMarco VG, Aroor AR \& Sowers JR (2014) The pathophysiology of hypertension in patients with obesity. Nat Rev Endocrinol 10, 364-376.

12. El Husseny MW, Mamdouh M, Shaban S, et al. (2017) Adipokines: potential therapeutic targets for vascular dysfunction in type ii diabetes mellitus and obesity. J Diabetes Res 2017, 8095926.

13. Matsuzawa Y (2006) Therapy Insight: adipocytokines in metabolic syndrome and related cardiovascular disease. Nat Clin Pract Cardiovasc Med 3, 35-42. 
14. Shibata R, Ouchi N, Ohashi K, et al. (2017) The role of adipokines in cardiovascular disease. J Cardiol 70, 329-334.

15. Kwon H \& Pessin JE (2013) Adipokines mediate inflammation and insulin resistance. Front Endocrinol (Lausanne) 4, 71.

16. Antuna-Puente B, Feve B, Fellahi S, et al. (2008) Adipokines: the missing link between insulin resistance and obesity. Diabet Metab 34, 2-11.

17. Dunmore SJ \& Brown JE (2013) The role of adipokines in beta-cell failure of type 2 diabetes. I Endocrinol 216, T37-T45.

18. Xiang AH, Kawakubo M, Trigo E, et al. (2010) Declining betacell compensation for insulin resistance in Hispanic women with recent gestational diabetes mellitus: association with changes in weight, adiponectin, and C-reactive protein. Diabet Care 33, 396-401.

19. Schwingshackl L \& Hoffmann G (2014) Mediterranean dietary pattern, inflammation and endothelial function: a systematic review and meta-analysis of intervention trials. Nutr Metab Cardiovasc Dis 24, 929-939.

20. Eichelmann F, Schwingshackl L, Fedirko V, et al. (2016) Effect of plant-based diets on obesity-related inflammatory profiles: a systematic review and meta-analysis of intervention trials. Obes Rev 17, 1067-1079.

21. Neale EP, Batterham MJ \& Tapsell LC (2016) Consumption of a healthy dietary pattern results in significant reductions in C-reactive protein levels in adults: a meta-analysis. Nutr Res 36, 391-401.

22. Shivappa N, Steck SE, Hurley TG, et al. (2014) Designing and developing a literature-derived, population-based dietary inflammatory index. Public Health Nutr 17, 1689-1696.

23. Tabung FK, Smith-Warner SA, Chavarro JE, et al. (2016) Development and validation of an empirical dietary inflammatory index. J Nutr 146, 1560-1570.

24. Watanabe RM, Allayee H, Xiang AH, et al. (2007) Transcription factor 7-like 2 (TCF7L2) is associated with gestational diabetes mellitus and interacts with adiposity to alter insulin secretion in Mexican Americans. Diabetes 56, 1481-1485.

25. Black MH, Fingerlin TE, Allayee H, et al. (2008) Evidence of interaction between PPARG2 and HNF4A contributing to variation in insulin sensitivity in Mexican Americans. Diabetes 57, 1048-1056

26. Li X, Allayee H, Xiang AH, et al. (2009) Variation in IGF2BP2 interacts with adiposity to alter insulin sensitivity in Mexican Americans. Obesity (Silver Spring) 17, 729-736.

27. Shu YH, Hartiala J, Xiang AH, et al. (2009) Evidence for sexspecific associations between variation in acid phosphatase locus 1 (ACP1) and insulin sensitivity in Mexican-Americans. J Clin Endocrinol Metab 94, 4094-4102.

28. Buchanan TA, Xiang AH, Kjos SL, et al. (1999) Antepartum predictors of the development of type 2 diabetes in Latino women 11-26 months after pregnancies complicated by gestational diabetes. Diabetes 48, 2430-2436.

29. Black MH, Watanabe RM, Trigo E, et al. (2013) High-fat diet is associated with obesity-mediated insulin resistance and beta-cell dysfunction in Mexican Americans. J Nutr 143, 479-485.

30. Willett WC, Sampson L, Stampfer MJ, et al. (1985) Reproducibility and validity of a semiquantitative food frequency questionnaire. Am J Epidemiol 122, 51-65.

31. Croghan CW \& Egeghy PP (2003) Methods of dealing with values below the limit of detection using SAS. http://analytics. ncsu.edu/sesug/2003/SD08-Croghan.pdf (accessed April 2017).

32. Knapp TR (1978) Canonical correlation analysis: a general parametric significance-testing system. Psychol Bull 85, 410-416.

33. Hotelling H (1936) Relations between two sets of variates. Biometrika 28, 321-377.
34. Therneau TM (2015) coxme: Mixed Effects Cox Models. R package version 2.2-5 2015. https://cran.r-project.org/web/ packages/coxme/index.html (accessed May 2017).

35. Degani A \& Shafto M (2006) Canonical correlation analysis: use of composite heliographs for representing multiple patterns. Diagrams 2006 - Diagrammatic Representation and Inference - 4th International Conference, Stanford, CA.

36. R Development Core Team (2016) R: A language and environment for statistical computing. Vienna, Austria: R Foundation for Statistical Computing.

37. Despres JP \& Lemieux I (2006) Abdominal obesity and metabolic syndrome. Nature $\mathbf{4 4 4}, 881-887$.

38. Schwartz MW, Seeley RJ, Zeltser LM, et al. (2017) Obesity pathogenesis: an endocrine society scientific statement. Endocr Rev 38, 1-30.

39. Rees K, Hartley L, Flowers N, et al. (2013) 'Mediterranean' dietary pattern for the primary prevention of cardiovascular disease. Cochrane Database Syst Rev, issue 8, CD009825.

40. Satija A, Bhupathiraju SN, Rimm EB, et al. (2016) Plant-based dietary patterns and incidence of type 2 diabetes in US men and women: results from three prospective cohort studies. PLoS Med 13, e1002039.

41. Shen J, Wilmot KA, Ghasemzadeh N, et al. (2015) Mediterranean dietary patterns and cardiovascular health. Annu Rev Nutr 35 , 425-449.

42. Obarzanek E, Sacks FM, Vollmer WM, et al. (2001) Effects on blood lipids of a blood pressure-lowering diet: The Dietary Approaches to Stop Hypertension (DASH) Trial. Am J Clin Nutr 74, 80-89.

43. Soltani S, Shirani F, Chitsazi MJ, et al. (2016) The effect of Dietary Approaches to Stop Hypertension (DASH) diet on weight and body composition in adults: a systematic review and meta-analysis of randomized controlled clinical trials. Obes Rev 17, 442-454.

44. Ogden CL, Kit BK, Carroll MD, et al. (2011) Consumption of sugar drinks in the United States, 2005-2008. NCHS Data Brief 71, 1-8.

45. Yngve A, Haapala I, Hodge A, et al. (2012) Making soft drinks the dietary version of the cigarette. Public Health Nutr 15, 1329-1330.

46. Malik VS, Pan A, Willett WC, et al. (2013) Sugar-sweetened beverages and weight gain in children and adults: a systematic review and meta-analysis. Am J Clin Nutr 98, 1084-1102.

47. Malik VS, Willett WC \& Hu FB (2009) Sugar-sweetened beverages and BMI in children and adolescents: reanalyses of a meta-analysis. Am J Clin Nutr 89, 438-439; author reply 9-40.

48. Malik VS, Popkin BM, Bray GA, et al. (2010) Sugar-sweetened beverages and risk of metabolic syndrome and type 2 diabetes: a meta-analysis. Diabet Care 33, 2477-2483.

49. Stanhope KL (2016) Sugar consumption, metabolic disease and obesity: The state of the controversy. Crit Rev Clin Lab Sci 53, 52-67.

50. Newby PK, Muller D, Hallfrisch J, et al. (2003) Dietary patterns and changes in body mass index and waist circumference in adults. Am J Clin Nutr 77, 1417-1425.

51. He K, Hu FB, Colditz GA, et al. (2004) Changes in intake of fruits and vegetables in relation to risk of obesity and weight gain among middle-aged women. Int J Obes Relat Metab Disord 28, 1569-1574.

52. Bell EA, Castellanos VH, Pelkman CL, et al. (1998) Energy density of foods affects energy intake in normalweight women. Am J Clin Nutr 67, 412-420.

53. Pereira MA \& Ludwig DS (2001) Dietary fiber and bodyweight regulation. Observations and mechanisms. Pediatr Clin North Am 48, 969-980.

54. Rangel-Huerta OD, Aguilera CM, Martin MV, et al. (2015) Normal or high polyphenol concentration in orange juice 
affects antioxidant activity, blood pressure, and body weight in obese or overweight adults. J Nutr 145, 1808-1816.

55. Deshmane SL, Kremlev S, Amini S, et al. (2009) Monocyte chemoattractant protein-1 (MCP-1): an overview. I Interferon Cytokine Res 29, 313-326.

56. Ouchi N, Higuchi A, Ohashi K, et al. (2010) Sfrp5 is an antiinflammatory adipokine that modulates metabolic dysfunction in obesity. Science 329, 454-457.

57. Chen K, Li F, Li J, et al. (2006) Induction of leptin resistance through direct interaction of C-reactive protein with leptin. Nat Med 12, 425-432.

58. Saltevo J, Vanhala M, Kautiainen H, et al. (2007) Levels of adiponectin, C-reactive protein and interleukin-1 receptor antagonist are associated with the relative change in body mass index between childhood and adulthood. Diab Vasc Dis Res 4, 328-331.

59. Yeh ET \& Willerson JT (2003) Coming of age of C-reactive protein: using inflammation markers in cardiology. Circulation 107, 370-371.

60. Freitas Lima LC, Braga VA, do Socorro de Franca Silva M, et al. (2015) Adipokines, diabetes and atherosclerosis: an inflammatory association. Front Physiol 6, 304.

61. Abella V, Scotece M, Conde J, et al. (2017) Leptin in the interplay of inflammation, metabolism and immune system disorders. Nat Rev Rheumatol 13, 100-109.

62. Koh KK, Park SM \& Quon MJ (2008) Leptin and cardiovascular disease: response to therapeutic interventions. Circulation 117, 3238-3249.

63. Bhupathiraju SN \& Tucker KL (2011) Greater variety in fruit and vegetable intake is associated with lower inflammation in Puerto Rican adults. Am J Clin Nutr 93, 37-46.

64. Fisk PS 2nd, Middaugh AL, Rhee YS, et al. (2011) Few favorable associations between fruit and vegetable intake and biomarkers for chronic disease risk in American adults. Nutr Res 31, 616-624

65. Esmaillzadeh A, Kimiagar M, Mehrabi Y, et al. (2006) Fruit and vegetable intakes, C-reactive protein, and the metabolic syndrome. Am J Clin Nutr 84, 1489-1497.

66. Gao X, Bermudez OI \& Tucker KL (2004) Plasma C-reactive protein and homocysteine concentrations are related to frequent fruit and vegetable intake in Hispanic and non-Hispanic white elders. J Nutr 134, 913-918.

67. Watzl B, Kulling SE, Moseneder J, et al. (2005) A 4-wk intervention with high intake of carotenoid-rich vegetables and fruit reduces plasma C-reactive protein in healthy, nonsmoking men. Am J Clin Nutr 82, 1052-1058.

68. Navarro P, de Dios O, Jois A, et al. (2017) Vegetable and fruit intakes are associated with hs-CRP levels in pre-pubertal girls. Nutrients 9, E224.

69. Mahoney SE \& Loprinzi PD (2014) Influence of flavonoid-rich fruit and vegetable intake on diabetic retinopathy and diabetesrelated biomarkers. J Diabet Complications 28, 767-771.

70. AlEssa HB, Ley SH, Rosner B, et al. (2016) High fiber and low starch intakes are associated with circulating intermediate biomarkers of type 2 diabetes among women. $J$ Nutr $\mathbf{1 4 6}$, 306-317

71. McCall DO, McGartland CP, McKinley MC, et al. (2011) The effect of increased dietary fruit and vegetable consumption on endothelial activation, inflammation and oxidative stress in hypertensive volunteers. Nutr Metab Cardiovasc Dis 21, 658-664.

72. McEvoy CT, Wallace IR, Hamill LL, et al. (2015) Increasing fruit and vegetable intake has no dose-response effect on conventional cardiovascular risk factors in overweight adults at high risk of developing cardiovascular disease. J Nutr $\mathbf{1 4 5}$ 1464-1471.
73. Nadeem N, Woodside JV, Neville CE, et al. (2014) Serum amyloid A-related inflammation is lowered by increased fruit and vegetable intake, while high-sensitive C-reactive protein, IL-6 and E-selectin remain unresponsive. Br J Nutr 112, 1129-1136.

74. Williams EJ, Baines KJ, Berthon BS, et al. (2017) Effects of an encapsulated fruit and vegetable juice concentrate on obesityinduced systemic inflammation: a randomised controlled trial. Nutrients 9, E116.

75. Heinonen MV, Laaksonen DE, Karhu T, et al. (2009) Effect of diet-induced weight loss on plasma apelin and cytokine levels in individuals with the metabolic syndrome. Nutr Metab Cardiovasc Dis 19, 626-633.

76. Kanda H, Tateya S, Tamori Y, et al. (2006) MCP-1 contributes to macrophage infiltration into adipose tissue, insulin resistance, and hepatic steatosis in obesity. J Clin Invest 116, $1494-1505$.

77. Rui L (2014) Energy metabolism in the liver. Compr Physiol 4 , 177-197.

78. Kim CS, Park HS, Kawada T, et al. (2006) Circulating levels of MCP-1 and IL- 8 are elevated in human obese subjects and associated with obesity-related parameters. Int J Obes (Lond) 30, $1347-1355$.

79. Sartipy P \& Loskutoff DJ (2003) Monocyte chemoattractant protein 1 in obesity and insulin resistance. Proc Natl Acad Sci U S A 100, 7265-7270.

80. Li YF, Chang YY, Huang HC, et al. (2015) Tomato juice supplementation in young women reduces inflammatory adipokine levels independently of body fat reduction. Nutrition 31, 691-696.

81. Cranford TL, Enos RT, Velazquez KT, et al. (2016) Role of MCP-1 on inflammatory processes and metabolic dysfunction following high-fat feedings in the $\mathrm{FVB} / \mathrm{N}$ strain. Int $J$ Obes (Lond) 40, 844-851.

82. Lin Y, Ye S, He Y, et al. (2018) Short-term insulin intensive therapy decreases MCP-1 and NF-kappa B expression of peripheral blood monocyte and the serum MCP-1 concentration in newly diagnosed type 2 diabetics. Arch Endocrinol Metab 62, 212-220.

83. Black MH, Shu YH, Wu J, et al. (2018) Longitudinal increases in adiposity contribute to worsening adipokine profile over time in Mexican Americans. Obesity (Silver Spring) 26, 703-712.

84. Helfer G \& Tups A (2016) Hypothalamic Wnt signalling and its role in energy balance regulation. J Neuroendocrinol $\mathbf{2 8}$, 12368.

85. Chen L, Zhao X, Liang G, et al. (2017) Recombinant SFRP5 protein significantly alleviated intrahepatic inflammation of nonalcoholic steatohepatitis. Nutr Metab (Lond) 14, 56.

86. Herder C, Carstensen M \& Ouwens DM (2013) Antiinflammatory cytokines and risk of type 2 diabetes. Diabetes Obes Metab 15, Suppl. 3, 39-50.

87. Schulte DM, Muller N, Neumann K, et al. (2012) Proinflammatory wnt5a and anti-inflammatory sFRP5 are differentially regulated by nutritional factors in obese human subjects. PLOS ONE 7, e32437.

88. Tan X, Wang X, Chu H, et al. (2014) SFRP5 correlates with obesity and metabolic syndrome and increases after weight loss in children. Clin Endocrinol (Oxf) 81, 363-369.

89. Fantuzzi G (2005) Adipose tissue, adipokines, and inflammation. J Allergy Clin Immunol 115, 911-919; quiz 20.

90. Wang X, Bao W, Liu J, et al. (2013) Inflammatory markers and risk of type 2 diabetes: a systematic review and meta-analysis. Diabet Care 36, 166-175.

91. Salvini S, Hunter DJ, Sampson L, et al. (1989) Food-based validation of a dietary questionnaire: the effects of week-toweek variation in food consumption. Int $J$ Epidemiol 18, $858-867$. 